\title{
Diffraction in the Context of the National Science Foundation (NSF) Carlos Murillo ${ }^{1}$ \\ ${ }^{1}$ NSF \\ cmurillo@nsf.gov
}

NSF has historically funded chemical instrumentation including X-ray diffractometers. A question that often raises is which Division is the best home for a proposal to be submitted to. We will explore such question for small chemical molecules, powder, protein or high-pressure studies. During the discussion we will provide background as to which are the major obstacles and common blunders in proposals from both from primarily undergraduate institutions and $\mathrm{PhD}$ granting institutions within the context of the Major Research Instrumentation (MRI) Program. In addition, we will discuss new advances and improvements coming to NSF's ChemMatCARS located at the Advanced Photon Source (APS) at Argonne National Laboratory. This facility, funded by NSF and managed by the Division of Chemistry, offers advanced synchrotron techniques free of charge.

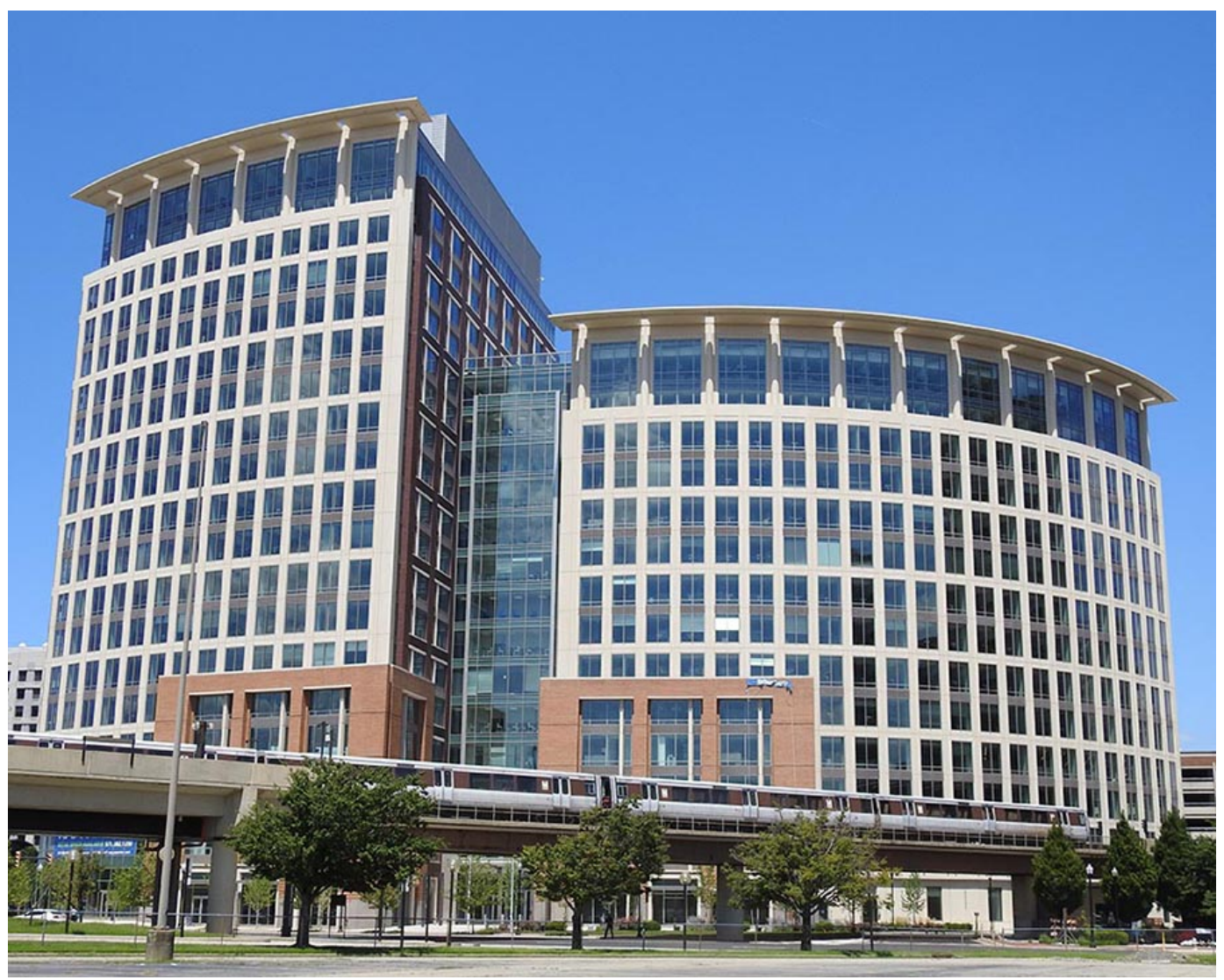

Figure 1 